Dubai

Journal
Dubai Med J 2019;2:153-157

DOI: $10.1159 / 000503992$
Received: June 6, 2019

Accepted: October 9, 2019

Published online: October 30, 2019

\title{
Implementation of World Health Organization Package of Essential Noncommunicable Disease Interventions for Cardiovascular Risk Management in Gaza/Palestine: A Retrospective Record Review Study
}

\author{
Rula AlHelo ${ }^{a} \quad$ Khamis Elessi ${ }^{b}$ \\ ${ }^{a}$ Noncommunicable Diseases Department, Primary Health Care, Ministry of Health, Gaza, Palestine; \\ ${ }^{b}$ Evidenced-Based Medicine Unit, Islamic University of Gaza, Gaza, Palestine
}

\section{Keywords}

Noncommunicable diseases · Cardiovascular risk · WHO

PEN protocol

\begin{abstract}
Background: Noncommunicable diseases (NCDs) are among the most common medical problems encountered in medical practice worldwide. The World Health Organization package of essential NCD (WHO PEN) interventions is a broad framework for integration of major NCDs into primary health care. These interventions include screening of diseases and their risk factors to prevent complications. WHO supports the adoption of WHO PEN into the Palestine Ministry of Health. PEN has been implemented in Gaza district since 2015, but only the protocols for cardiovascular disease and diabetes were selected for implementation. This study may help us to detect the impact of implementing PEN. Methods: A retrospective record review study was conducted using a random sample of 143 files from a total of 280 registered NCD patients who were followed up for 2 years at Alsourani clinic. Data were extracted manually from the medical records with the completion of a data sheet. Results: The study showed that most of the population was female ( $n=86$,
\end{abstract}

$60.1 \%)$. The mean age was $58.8 \pm 11.7$ years. The vast majority were living a sedentary lifestyle ( $n=141,98.6 \%)$. Patients with one or more comorbidities were also tabulated according to the presence of each comorbidity among all subjects: hypertension ( $n=92,64.3 \%$ ), any diabetes ( $n=90,62.9 \%$ ), type 2 diabetes ( $n=86,60.1 \%)$, type 1 diabetes $(n=4,2.8 \%)$, dyslipidemia ( $n=41,28.7 \%$ ), neuropathy ( $n=27,18.9 \%$ ), ischemic heart disease $(n=20,14 \%)$, cerebrovascular disease ( $n=4,2.8 \%)$, retinopathy $(n=8,5.6 \%)$, mental illness $(n=4$, $2.8 \%)$, nephropathy ( $n=3,2.1 \%)$, cancer $(n=2,1.4 \%)$, and respiratory diseases $(n=1,0.7 \%)$. There was no statistically significant difference between pre- and post-parameter interventions for systolic and diastolic blood pressure, waist circumference, weight, body mass index, and cholesterol, as well as for tobacco use and cardiovascular risk $(p>0.05)$. In contrast, fasting blood sugar was lower, with a statistically significant difference ( $p=0.049)$. Conclusion: NCDs in Palestine are a major health problem. Sustainability of WHO PEN interventions is recommended with insurance to support the needs of NCD patients and home visits. NCD interventions are compromised due to health care system gaps at primary care level. Therefore, we need strengthening of the primary health care system.

(c) 2019 The Author(s)

Published by S. Karger AG, Basel

\begin{tabular}{ll}
\hline KARGER & $\begin{array}{l}\text { ( } 2019 \text { The Author(s) Karger } \\
\text { Published by S. Karger AG, Basel Open caccess }\end{array}$ \\
E-Mail karger@karger.com & $\begin{array}{l}\text { This article is licensed under the Creative Commons Attribution- } \\
\text { NonCommercial-NoDerivatives 4.0 International License (CC BY- } \\
\text { NC-ND) (http://www.karger.com/Services/OpenAccessLicense). } \\
\text { Wwwage and distribution for commercial purposes as well as any dis- } \\
\text { tribution of modified material requires written permission. }\end{array}$
\end{tabular}

Dr. Rula AlHelo

Chief of Training, Noncommunicable Diseases Department Primary Health Care, Ministry of Health, AlWehdah Street Gaza 79702 (Palestine)

E-Mail dr_rula@hotmail.com 


\section{Introduction}

Currently, noncommunicable diseases (NCDs) are among the most common medical problems encountered in medical practice both in developed and developing countries. They are chronic diseases that result from a constellation of risk factors (genetic, environmental). They compromise mainly cardiovascular diseases, cancer, diabetes, and chronic lung diseases and associated risk factors $[1,2]$. NCDs are preventable and can be treated to reduce morbidity and mortality [3].

The World Health Organization package of essential NCD (WHO PEN) interventions is a broad framework for integration of major NCDs into primary health care (PHC). These interventions include screening of diseases and their risk factors to prevent complications. It involves five actions, step by step, including early detection of complications to reduce devastating consequences (e.g., annual fundus examination of patients with diabetes to detect retinopathy in early stages and prevent blindness) $[1,4]$.

NCDs kill 38 million people each year, which is equivalent to $70 \%$ of all deaths globally, including 16 million premature deaths (before the age of 70 ), $80 \%$ of which occur in low- and middle-income countries [1].

NCDs in Palestine are a major health problem $[5,6]$. Cardiovascular diseases accounted for $22.4 \%$ of all deaths in Palestine in 2011 [7]. A survey was conducted in 2010-2011 regarding risk factors. The risk factors involved in the survey were current daily smoking, less than 5 servings of fruits and vegetables per day, low level of activity, overweight, raised blood pressure (BP), raised fasting blood sugar (FBS), and raised total cholesterol. In Gaza, the percentage with none of the above first five risk factors was $0.2 \%$; the percentage with three or more, aged $15-44$ years, was $48.1 \%$, aged $45-64$ years $83.7 \%$, and aged $15-64$ years $54.6 \%$ [8]. WHO supports the adoption of WHO PEN into the Palestine Ministry of Health. In Gaza, in 2013, PHC implemented the interventions in four districts (implemented in Gaza district in 2015); only the protocols for cardiovascular disease and diabetes were selected for implementation. The Ministry of Health adopted WHO PEN to decrease the financial burden of NCDs and improve health outcomes. The objective of this study was to evaluate the impact of these interventions in reducing cardiovascular disease risk as these interventions are newly implemented.

\section{Materials and Methods}

This study is a retrospective evaluation of the implementation of the PEN over a period of 2 years. The study was conducted using a random sample of 143 files from a total of 280 registered patients who were followed up for 2 years (April 2015-March 2017) at Alsourani clinic, Gaza, Palestine. Patients included those who had renewed their files according to PEN protocol and were followed up by a team trained in the protocol. Patients were excluded from the study if their medical records contained insufficient data.

Data were extracted manually from the medical records with completion of a data sheet. Demographic and behavioral data (age, gender, tobacco use, engaged in more than $30 \mathrm{~min}$ of physical activity at least 5 days a week, pre-existing history of NCD) were reviewed. The following data were recorded: measured $\mathrm{BP}$ (raised if systolic BP $[\mathrm{SBP}] \geq 140$ and/or diastolic BP $[\mathrm{DBP}] \geq 90 \mathrm{~mm} \mathrm{Hg}$ or currently on medication for raised BP), body mass index (BMI) (overweight if $\geq 25 \mathrm{~kg} / \mathrm{m}^{2}$ ), waist circumference (WC) (increased if $\geq 90 \mathrm{~cm}$ in women or $\geq 100 \mathrm{~cm}$ in men), laboratory results of FBS (raised if plasma venous value $\geq 126 \mathrm{mg} / \mathrm{dL}$ or currently on medication for raised blood glucose) and cholesterol (raised if $\geq 200 \mathrm{mg}$ / $\mathrm{dL}$ or currently on medication for raised cholesterol), and predicted 10-year cardiovascular risk (CVR) (using the WHO/ISH risk charts relevant to the WHO subregion) before and after implementation of PEN interventions. Definitions were used to describe the studied population, while the outcome measure was change in parameters from baseline to 2 years.

Data management and analysis were conducted using the SPSS program version 23.0. Continuous variables are presented as means $\pm S D$, whereas categorical variables are presented as numbers and percentages. The paired $t$ test was used to test for differences between quantitative variables, while the McNemar test was used to compare between proportions. A $p$ value of $<0.05$ was considered to be statistically significant.

\section{Results}

Table 1 shows demographic data and pre-existing history of NCDs. The total study population included 143 cases of NCD patients, of whom 57 were male patients $(39.9 \%)$ and 86 were female $(60.1 \%)$. The mean age of the studied population was $58.8 \pm 11.7$ years. The vast majority of our study sample confirmed that they were living a sedentary lifestyle (98.6\%) and only 2 subjects claimed that they were practicing regular physical activity. At the end of the study, patients with one or more comorbidities were also tabulated according to the presence of each co-morbidity among all subjects: hypertension $(n=92$, $64.3 \%)$, any diabetes $(n=90,62.9 \%)$, type 2 diabetes $(n=$ $86,60.1 \%)$, type 1 diabetes $(n=4,2.8 \%)$, dyslipidemia ( $n=41,28.7 \%)$, neuropathy $(n=27,18.9 \%)$, ischemic heart disease $(n=20,14 \%)$, cerebrovascular disease $(n=$ $4,2.8 \%)$, retinopathy $(n=8,5.6 \%)$, mental illness $(n=4$, 
Table 1. Descriptive characteristics of the studied population

\begin{tabular}{|c|c|c|c|c|c|c|c|c|}
\hline Age, years & & $58.8 \pm 11.7$ & & & & & & \\
\hline \multirow[t]{2}{*}{ Gender } & male & 57 (39.9) & DM1 & yes & $4(2.8)$ & Nephropathy & yes & $3(2.1)$ \\
\hline & female & $86(60.1)$ & & no & $139(97.2)$ & & no & 140 (97.9) \\
\hline \multirow[t]{2}{*}{ Activity } & yes & $2(1.4)$ & DM2 & yes & $86(60.1)$ & ESRD & yes & $0(0)$ \\
\hline & no & $141(98.6)$ & & no & $57(39.9)$ & & no & $143(100.0)$ \\
\hline \multirow[t]{2}{*}{ HTN } & yes & $92(64.3)$ & Dyslipidemia & yes & $41(28.7)$ & Neuropathy & yes & $27(18.9)$ \\
\hline & no & $51(35.7)$ & & no & $102(71.3)$ & & no & $116(81.1)$ \\
\hline \multirow[t]{2}{*}{ IHD } & yes & $20(14.0)$ & Psychiatry & yes & $4(2.8)$ & Diabetic foot & yes & $1(0.7)$ \\
\hline & no & $123(86.0)$ & & no & $139(97.2)$ & & no & $142(99.3)$ \\
\hline \multirow[t]{2}{*}{ CVA } & yes & $4(2.8)$ & Cancer & yes & $2(1.4)$ & Amputation & no & $143(100.0)$ \\
\hline & no & $139(97.2)$ & & no & $141(98.6)$ & & yes & $0(0)$ \\
\hline \multirow[t]{2}{*}{ Respiratory } & yes & $1(0.7)$ & Retinopathy & yes & $8(5.6)$ & & & \\
\hline & no & $142(99.3)$ & & no & $135(94.4)$ & & & \\
\hline
\end{tabular}

Data are presented as $n(\%)$ or mean \pm SD as appropriate. HTN, hypertension; IHD, ischemic heart disease; CVA, cerebrovascular accident; DM, diabetes mellitus; ESRD, end-stage renal disease.

$2.8 \%)$, nephropathy $(n=3,2.1 \%)$, cancer $(n=2,1.4 \%)$, and respiratory diseases $(n=1,0.7 \%)$. One case only had diabetic foot with no amputations and there were no cases with ESRD.

Table 2 shows the effects of PEN implementation on SBP, DBP, FBS, WC, WT, BMI, and cholesterol. There was no statistically significant difference between preand post-parameter interventions for SBP, DBP, WC, WT, BMI, and cholesterol $(p>0.05)$. In contrast, FBS was lower, with a statistically significant difference in postparameter $(133.2 \pm 62.5)$ compared to pre-parameter (144.4 \pm 65.4 ) interventions ( $p=0.049)$.

Table 3 shows the effects of PEN implementation on tobacco and CVR. There was no statistically significant difference between pre- and post-parameter interventions for tobacco and CVR ( $p$ value $>0.05$ )

\section{Discussion}

Based on our study findings, we cannot prove any association of SBP, DBP, WC, BMI, cholesterol level, tobacco use, and predicted CVR before and after WHO PEN interventions. In contrast, FBS reduced over the 2 years of interventions.

Inconsistent with our results, a study in the Democratic People's Republic of Korea, where WHO PEN interventions were implemented, found a significant reduction in risk factors and predicted CVR over a 1-year period [9]. The availability of free medicines and equipment with the system of household doctors may have helped in that improvement. Similar findings were ob-

Noncommunicable Diseases in Primary

Health Care
Table 2. Pre- and post-parameter intervention among the studied population

\begin{tabular}{lccrc}
\hline & \multicolumn{2}{l}{ Intervention $^{1}$} & \multirow{2}{*}{$t$ value } & $p$ value \\
\cline { 2 - 3 } & pre & post & & \\
\hline SBP & $120.5 \pm 11.9$ & $122.4 \pm 12.3$ & 1.960 & 0.052 \\
DBP & $78.9 \pm 7.6$ & $80 \pm 8$ & 1.775 & 0.078 \\
WC & $107 \pm 14.5$ & $106.4 \pm 11.5$ & -0.826 & 0.410 \\
WT & $86.1 \pm 16.1$ & $85.6 \pm 16$ & -1.446 & 0.150 \\
BMI & $32.6 \pm 5.5$ & $32.4 \pm 5.6$ & -1.518 & 0.131 \\
FBS & $144.4 \pm 65.4$ & $133.2 \pm 62.5$ & -1.984 & 0.049 \\
Cholesterol & $183.6 \pm 37.3$ & $182.5 \pm 45.5$ & -0.304 & 0.762
\end{tabular}

SBP, systolic blood pressure; DBP, diastolic blood pressure; WC, waist circumference; WT, weight; BMI, body mass index; FBS, fasting blood sugar.

${ }^{1}$ WHO PEN intervention.

Table 3. Pre- and post-parameter intervention among the studied population

\begin{tabular}{clccc}
\hline & & \multicolumn{2}{c}{ Intervention } & \multirow{2}{*}{$p$ value } \\
\cline { 3 - 4 } & & pre & post & \\
\hline \multirow{2}{*}{ Tobacco } & yes & $8(5.6)$ & $6(4.2)$ & \multirow{2}{*}{0.584} \\
& no & $135(94.4)$ & $137(95.8)$ & \\
CVR & $<10 \%$ & $107(74.8)$ & $110(76.9)$ & \multirow{2}{*}{0.927} \\
& $10-<20 \%$ & $8(5.6)$ & $7(4.9)$ & \\
& $20-<30 \%$ & $2(1.4)$ & $1(0.7)$ & \\
& $30-<40 \%$ & $26(18.2)$ & $25(17.5)$ & \\
& & & &
\end{tabular}

CVR, cardiovascular risk. 
served in the evaluation of the Bhutan PEN project [10]. Gaza faced many challenges in the implementation of WHO PEN due to a defect in the sustainability of medicines, testing devices, and strips (e.g., urinary dipstick) throughout the year. Affordability of essential medicines as an important component of universal health coverage is crucial to promoting health and achieving sustainable development [11]. Indeed, lack of home visits to patients who cannot visit the clinic regularly is another problem. It was also noticed that poor socioeconomic status of NCD patients affected the outcome and health financing. Obstacles of continuing care should be identified to improve the effectiveness of NCD interventions [12].

Additionally, in Ljubljana, three successive surveys were carried out to measure 12 years of BP dynamics in adults after an integrated NCD intervention program. No significant reduction was found in the first two surveys but in the third survey after 12 years a significant reduction was reached [13]. Although we did not find a significant association over short-term effects ( 2 years), the positive outcome of WHO PEN is that trained health care workers had an increased awareness of NCD management, referral criteria, and use of monitoring tools. In addition, PEN served in the documentation of risk factors (e.g., WC) and calculation of CVR and took action according to the score. A PHC approach is essential to address NCDs $[14,15]$. A clinical audit for WHO PEN implementation was done in the Salfit district, Palestine. The audit showed general commitment of the staff to the protocol with some concerns and identified areas which need more training and supervision [16]. Therefore, use of an audit is an effective technique to assess the quality of services and improve it. Accordingly, strengthening the $\mathrm{PHC}$ system is a key challenge to improving the delivery of cost-effective interventions [17].

\section{Conclusion}

NCDs constitute a major health problem in Palestine. Sustainability of WHO PEN interventions is recommended with insurance to support the needs of NCD patients and home visits. NCD interventions are compromised due to gaps in the health care system at primary care level. Therefore, we need strengthening of the PHC system.

\section{Acknowledgments}

We thank the staff of the Palestine Ministry of Health, the Noncommunicable Diseases Department, the Primary Health Care Directorate, and G.D. of Human Resources Development.

\section{Statement of Ethics}

Ethical approval was obtained to check patient records for the purpose of the study.

\section{Disclosure Statement}

The authors have no conflicts of interest to declare.

\section{Funding Sources}

No funding was received for this study.

\section{Author Contributions}

R.A. developed the research question, initiated and conceptualized the study idea, reviewed previous studies and reports, and drafted the study proposal. K.E. advised on the methodology and statistical analysis section, and interpretation of results. R.A. wrote the final document and revised it frequently after input from K.E. Both authors reviewed and agreed on the final version of the manuscript.

\section{References}

1 World Health Organization. WHO global status report on noncommunicable diseases. 2010. Available from: http://www.who.int/ ncds/management.

2 Global action plan for the prevention and control of NCDs 2013-2020. Available from: https://www.who.int/nmh/events/ncd_action_plan.
3 Prabhakaran D, Anand S, Gaziano TA, et al., editors. Cardiovascular, respiratory, and related disorders. 3rd ed. Washington (DC): The International Bank for Reconstruction and Development/The World Bank; 2017.

4 World Health Organization. Package of Essential Noncommunicable (PEN) disease interventions for primary health care in low-resource settings. 2010. http://apps.who.int/iris/ bitstream/10665/44260/1/9789241598996_ eng.pdf.
5 Husseini A, Abu-Rmeileh NM, Mikki N, Ramahi TM, Ghosh HA, Barghuthi N, et al. Cardiovascular diseases, diabetes mellitus, and cancer in the occupied Palestinian territory. Lancet. 2009 Mar;373(9668):1041-9.

6 Jamee Shahwan A, Abed Y, et al. (2019). Epidemiology of coronary artery disease and stroke and associated risk factors in Gaza community - Palestine. PLoS One. 2019 Jan; 14(1):e0211131. 
7 Ministry of Health. Health report Palestine. 2011 [cited 2013 February 26]. Available from: http://www.moh.ps/attach/440.pdf.

8 WHO EMRO|STEPwise surveillance 20102011. Available from: https://www.who.int/ ncds/surveillance/steps/opt/en/.

9 Choe Suk Hyon, Kim Yong Nam, et al. Package of essential noncommunicable disease (PEN) interventions in primary health-care settings in the Democratic People's Republic of Korea: a feasibility study. WHO South-East Asia J Public Health. 2017 Sep;6(2):69-73.

10 Wangchuk D, Virdi NK, Garg R, Mendis S, Nair N, Wangchuk D, et al. Package of essential noncommunicable disease (PEN) interventions in primary health-care settings of Bhutan: a performance assessment study. WHO South-East Asia J Public Health. 2014 Apr-Jun;3(2):154-60.
11 Jarvis JD, Woods $\mathrm{H}$, et al. Selection of WHOrecommended essential medicines for noncommunicable diseases on National Essential Medicines Lists. PLoS One. 2019 Aug 9; 14(8)):e0220781.

12 Mendis S, Al Bashir I, Dissanayake L, Varghese C, Fadhil I, Marhe E, et al. Gaps in capacity in primary care in low-resource settings for implementation of essential noncommunicable disease interventions. Int $\mathrm{J}$ Hypertens. 2012;2012(Nov):584041.

13 Bulc M, Fras Z, Zaletel-Kragelj L. Twelve-year blood pressure dynamics in adults in Ljubljana area, Slovenia: contribution of WHO Countrywide Integrated Noncommunicable Diseases Intervention Program. Croat Med J. 2006 Jun;47(3):469-77.

14 Mendis S, Johnston SC, Fan W, Oladapo O, Cameron A, Faramawi MF. Cardiovascular risk management and its impact on hypertension control in primary care in low-resource settings: a cluster-randomized trial. Bull World Health Organ. 2010 Jun;88(6):412-9.
15 World Health Organization. Declaration of Alma-Ata. 1978. Available from: http://www. who.int/publications/almaata_declaration_ en.pdf.

16 Dweakat R, Barghouthi N, Bouziyeh Y, et al. Clinical audit to assess quality of service in a newly implemented NCD programme: a cross-sectional survey to review a pilot implementation of the WHO PEN approach in Salfit district, occupied Palestinian territory. Lancet. 2017;390(suppl 1):S28.

17 Lewin S, Lavis JN, Oxman AD, Bastías G, Chopra M, Ciapponi A, et al. Supporting the delivery of cost-effective interventions in primary health-care systems in low-income and middle-income countries: an overview of systematic reviews. Lancet. 2008 Sep;372(9642): 928-39. 